\title{
Altered Expression of the Transcription Factor Mef2c during Retinal Degeneration in Rpe $65^{-/-}$Mice
}

\author{
Pascal Escher, ${ }^{1,2}$ Daniel F. Schorderet, ${ }^{1,2,3}$ and Sandra Cottet ${ }^{1,2}$
}

Purpose. To investigate the role of the myocyte enhancer factor 2 (Mef2) transcription factor family in retinal diseases, Mef $2 c$ expression was assessed during retinal degeneration in the Rpe $65^{-1-}$ mouse model of Leber's congenital amaurosis (LCA). Mef2c-dependent expression of photoreceptor-specific genes was further addressed.

Methods. Expression of Mef2 members was analyzed by oligonucleotide microarray, quantitative PCR (qPCR), and in situ hybridization. Mef2c-dependent transcriptional activity was assayed by luciferase assay in HEK293T cells.

Results. Mef2c was the only Mef2 member markedly downregulated during retinal degeneration in $R p e 65^{-/-}$mice. Mef2c mRNA level was decreased by more than 2 -fold at 2 and 4 months and by 3.5-fold at 6 months in retinas of $R p e 65^{-/-}$mice. Downregulation of Mef2c at the protein level was confirmed in Rpe $65^{-/-}$retinas. The decrease in Mef2c mRNA levels in the developing $R p e 65^{-/-}$retinas from postnatal day $(\mathrm{P}) 13$ onward was concomitant with the decreased expression of the rodspecific transcription factors $\mathrm{Nrl}$ and $\mathrm{Nr} 2 \mathrm{e} 3$. Nrl was further shown to drive Mef2c transcriptional activity, supporting a physiological role for Mef2c in the retina. In addition, Mef2c appeared to act as a transcriptional repressor of its own expression and the expression of the retina-specific retinal G-protein coupled receptor (Rgr), rhodopsin, and M-opsin genes.

Conclusions. These findings highlight the early altered regulation of the rod-specific transcriptional network in Rpe65related disease. They also indicate that Mef2c may act as a novel transcription factor involved in the development and the maintenance of photoreceptor cells. (Invest Ophthalmol Vis Sci. 2011;52:5933-5940) DOI:10.1167/iovs.10-6978

M utations in the gene encoding the RPE-specific protein 65 $\mathrm{kDa}$ (RPE65) account for approximately $10 \%$ of cases of Leber's congenital amaurosis (LCA). ${ }^{1}$ The hallmarks of LCA pathogenesis are early and severe rod-cone dystrophy. ${ }^{2}$ Affected patients experience the degeneration of photoreceptor cells associated with congenital nystagmus, profound visual deficiency, night blindness, and reduced or nondetectable ERG.

The iron (II)-dependent isomerohydrolase RPE65 directs the synthesis of the 11-cis retinol necessary for light-induced

From the ${ }^{1}$ Institute for Research in Ophthalmology, Sion, Switzerland; ${ }^{2}$ Department of Ophthalmology, University of Lausanne, Lausanne, Switzerland; and ${ }^{3}$ School of Life Sciences, Federal Institute of Technology, Lausanne, Switzerland.

Supported by The Swiss National Science Foundation Grants 31003A-118336 (SC and DFS) and 31003A-122269 (PE and DFS).

Submitted for publication December 1, 2010; revised May 23 and June 10, 2011; accepted June 14, 2011

Disclosure: P. Escher, None; D.F. Schorderet, None; S. Cottet, None

Corresponding author: Sandra Cottet, Avenue Grand-Champsec 64, 1950 Sion, Switzerland; sandra.cottet@irovision.ch. phototransduction mediated by rod and cone opsins. ${ }^{3-5}$ $R$ pe $65^{-/-}$mice exhibit defects in retinal morphology, function, and biochemistry resembling those seen in human LCA. ${ }^{6,7}$ In the absence of RPE65, all-trans-retinyl esters accumulate in lipid droplets within the RPE, reflecting defects in the visual cycle in these cells. ${ }^{6}$ Rpe $65^{-1-}$ mice lack the 11-cis retinal chromophore, do not generate adequate levels of visual pigment, and have severely depressed light- and dark-adapted ERG responses. ${ }^{6-8}$ Unliganded rod opsin triggers light-independent, constitutive activation of residual phototransduction cascade leading to photoreceptor cell death ${ }^{9}$ through a Bax-related pathway. ${ }^{10}$

The myocyte enhancer factor 2 (Mef2) family of transcription factors comprises four gene products in mammals, Mef2a-d. ${ }^{11}$ Mef2 proteins (Mef2s) function as central regulators of diverse developmental programs controlling cell differentiation and organogenesis, and are especially involved in muscle and neuronal differentiation (reviewed by Potthoff and Olson ${ }^{12}$ ). Mef2s contain in the $n$ terminus the MADS (MCM1-agamous-deficiensserum response factor)-box and the Mef2 domain responsible for DNA binding, dimerization, and cofactor recruitment. These two regions are highly conserved, with around 95\% amino acid similarity among the vertebrate Mef2 factors. The $\mathrm{C}$ terminus of Mef2s contains an evolutionarily divergent transcriptional activation domain subjected to a complex pattern of alternative splicing. ${ }^{1,12}$ Mef2 homo- or heterodimers bind to the consensus DNA regulatory sequence (C/Y)TA(A/ $\mathrm{T})_{4} \mathrm{TA}(\mathrm{G} / \mathrm{R}){ }^{11,13}$ The transcriptional activity of Mef2s is stimulated by mitogen-activated protein kinase signaling. ${ }^{12}$ Calciumdependent signals also activate Mef2s by stimulating calciumdependent kinases that phosphorylate class II histone deacetylases, thereby promoting their dissociation from Mef2 and derepressing Mef2 target genes. In addition, Mef2s recruit numerous cofactors to drive specific cell differentiation.

Mef2s were originally described as muscle-enriched transcription factors in differentiated myotubes and were recognized as key regulators of cardiac myogenesis and morphogenesis. ${ }^{11}$ For instance, mice with a targeted disruption of Mef $2 c$ $\left(M e f 2 c^{-/-}\right.$mice) died early during embryonic development because the heart tube did not undergo looping morphogenesis and the right ventricle did not form. ${ }^{14}$ During skeletal muscle development, Mef2s cooperate and strongly potentiate the transcriptional activity of the myogenic basic helix-loophelix (bHLH) proteins MyoD, Myf5, myogenin, and MRF4. ${ }^{11}$ In vertebrates, the myogenic bHLH transcription factors directly activate Mef2c expression by binding to a skeletal musclespecific enhancer located immediately upstream of the first untranslated exon of mouse Mef2c, which in mice is $71 \mathrm{~kb}$ upstream of the first translated exon. ${ }^{15,16}$ In addition to striated muscles, Mef2s are highly expressed in several brain regions. ${ }^{17-19}$ In this organ, time-regulated expression of Mef2 proteins is consistent with their role in neuronal differentiation and maintenance (reviewed by Heidenreich and Linseman ${ }^{20}$ ).

Mef2c is robustly expressed by cortical neurons and represents the predominant Mef2 member in the cerebral cortex. ${ }^{21}$ 
Embryonic conditional knock-out of Mef2c in nestin-expressing neural stem/progenitor cells resulted in impaired differentiation of postmitotic neurons associated with neuronal defects and severe behavioral deficits. ${ }^{22}$ These data further supported a crucial role of Mef2c in the neocortex with respect to neuron development, distribution, and electrical activity. ${ }^{19,21,23}$ Finally, human MEF2C haploinsufficiency caused by a small deletion within the MEF2C gene has been associated with severe mental retardation and cerebral malformation. ${ }^{24-27}$

The observation that Mef2c levels were decreased at the transcriptional level in several models of retinal degeneration-the $R p e 65^{-1-}, 28$ the $\mathrm{Rho}^{-/-}, 29$ the $\mathrm{Crx}^{-1-30}$ and the $\mathrm{Nrl}^{-/-31}$ mouse-prompted us to further investigate Mef2c in the retina.

\section{Methods}

\section{Mouse Lines and Genotyping}

These studies adhered to the ARVO statement for the use of animals in ophthalmic and vision research and were approved by the Veterinary Service of the State of Valais (Switzerland). Wild-type C57BL/6 mice (WT) were purchased from Charles River Laboratories (Les Oncins, France). Rpe $65^{-/-}$mice are on a C57BL/6 genetic background (from T. Michael Redmond, National Institutes of Health, Bethesda, MD). The genotype of the mice was determined by PCR analysis with genomic DNA isolated from tail tissue. Animals were kept in a 12-hour light/dark cycle with unlimited access to food and water.

\section{Tissue Isolation and RNA Extraction}

Age-matched animals were killed by cervical dislocation. Retinas from each mouse strain were quickly isolated in RNA stabilization reagent (RNAlater; Ambion, Huntingdon, UK) before being transferred in total RNA isolation reagent (TRIzol; Invitrogen, Basel, Switzerland) and stored at $-80^{\circ} \mathrm{C}$ until RNA extraction. Total RNA was extracted according to manufacturer's instructions, and the amount of total RNA was determined by a quantitation assay (RiboGreen; Invitrogen).

\section{Oligonucleotide Microarray}

Oligonucleotide microarray was previously described in detail. ${ }^{28}$ Briefly, $1 \mu \mathrm{g}$ of total RNA was used to generate double-stranded cDNA used as a template for biotinylated cRNA synthesis using a commercially available kit (Affymetrix GeneChip Expression 3'-Amplification Kit for IVT Labeling Kit; Affymetrix, Santa Clara, CA). Next, $20 \mu \mathrm{g}$ of target CRNA were fragmented and hybridized on an array (Affymetrix Mouse Genome 4302.0 GeneChips; Affymetrix), and washed chips were scanned on a scanner (Affymetrix GeneChip Scanner 3000 using GCOS software; Affymetrix). Data normalization was performed using the Robust Multi-Array Analysis algorithm (RMA) as implemented in specific commercially available software (GeneSpring, version 7.2; Agilent Technologies, Waldbronn, Germany). Three mice were used for each condition studied.

\section{Quantitative PCR Analysis}

The equivalent of 50 ng of total RNA was used for PCR amplification using a PCR reagent $(2 \times$ brilliant SYBR Green QPCR Master Mix; Agilent) with either $125 \mathrm{nM}$ (Mef2a, Mef2d, Nr2e3, and Nrl), $250 \mathrm{nM}$ (Mef2b or Mef2c), or $400 \mathrm{nM}$ (Rl8) forward and reverse primer pairs. Real-time PCR was performed in triplicate in a PCR system (Mx3000P; Agilent) with the following cycling conditions: 40 cycles of denaturation at $95^{\circ} \mathrm{C}$ for 30 seconds, annealing at $55^{\circ} \mathrm{C}$ (except for $M e f 2 b$ at $65^{\circ} \mathrm{C}$ ) for 30 seconds, and extension at $72^{\circ} \mathrm{C}$ for 60 seconds. Quantitative values were obtained by the cycle number (Ct value) reflecting the point at which fluorescence starts to increase above background at a fixed threshold level. Values obtained for the target genes were normalized with the housekeeping ribosomal protein L8 (Rl8) gene. Three to four mouse retinas were used for each condition studied. Primer sequences are shown in Table 1.

\section{In Situ Hybridization}

Eyes were enucleated and fixed for 30 minutes in ice cold $4 \%$ paraformaldehyde $(\mathrm{PFA}) / 1 \times$ PBS-diethylpyrocarbonate (DEPC) and then transferred to a $30 \%$ sucrose $/ \mathrm{H}_{2} \mathrm{O}-\mathrm{DEPC}$ solution overnight at $4{ }^{\circ} \mathrm{C}$. Fixed and cryopreserved eyes were embedded in Yazzulla (30\% albumin/3\% gelatin in PBS-DEPC) and cut into 10- $\mu \mathrm{m}$ thick cryosections using a cryostat (CM1900; Leica Microsystems, Heerbrugg, Switzerland). Eye sections were thawed and mounted on reagent (Vectabond; Vector Laboratories, Burlingame, VT)-treated glass slides (SuperFrost Plus; Menzel Gläser, Braunschweig, Germany). PCR amplified Mef $2 c$ cDNA fragment (nucleotides 406 to 810 from the mouse Mef2c coding sequence) was subcloned into a vector (pGEM-T Easy Vector; Promega, Madison, WI) according to the manufacturer's protocol. Blast analysis of the sequence used for generating the Mef2c probe showed no similarity with the other Mef2 genes. Plasmids were linearized with SpeI and ApaI restriction enzymes to obtain a template sequence for either the sense or antisense RNA probes. Digoxygenin (DIG)-labeled antisense and sense cRNA probes were synthesized by in vitro transcription of $1 \mu \mathrm{g}$ of linearized DNA with either T7 or Sp6 RNA polymerases (DIG RNA labeling mix; Roche, Rotkreuz, Switzerland) according to the manufacturer's protocol. DIG-labeled sense and antisense probes were tested by immunodot blotting and equal amounts of probe (1/100 dilution) were used in the experiment. In situ hybridizations were performed at a hybridization temperature of $48^{\circ} \mathrm{C}$ as previously described. ${ }^{32}$ Slides were then washed twice in $0.1 \times$ SSC at $65^{\circ} \mathrm{C}$ for 30 minutes and then incubated for 2 hours at room temperature (RT) with anti-DIG antibodies alkaline phosphatase (AP) conjugated (Roche) diluted at 1/5,000. Color staining was developed by overnight incubation with NBT/BCIP (Roche) in the dark at RT. The reaction was stopped by adding TE (1/10), followed by a final wash in 95\% EtOH for 45 minutes Eyes from three mice were analyzed for each genotype.

\section{Subcellular Fractionation}

Cytosolic and nuclear fractions were prepared from adult WT and Rpe $65^{-/-}$retinas using extraction reagents (NE-PER Nuclear and Cytoplasmic Extraction Reagents; Thermo Fisher Scientific, Lausanne, Swit-

TABle 1. Primer Sequences for Real-Time PCR Analysis

\begin{tabular}{|c|c|c|c|}
\hline Gene & Forward Primer $\left(5^{\prime}-3^{\prime}\right)$ & Reverse Primer $\left(5^{\prime}-3^{\prime}\right)$ & $\begin{array}{c}\text { GenBank } \\
\text { Accession Number }\end{array}$ \\
\hline $\operatorname{Mef} 2 a$ & AGCAGCACCATCTAGGACAA & CTGCTGTTGGAAGCCTGATG & U30823 \\
\hline $\operatorname{Mef} 2 b$ & GCAACGCCTCTTCCAGTATG & AGTGTCCTCAGCAGCTTCTC & D50311 \\
\hline $\operatorname{Mef} 2 c$ & AGATCTGACATCCGGTGCAG & TCTTGTTCAGGTTACCAGGT & NM_025282 \\
\hline $\operatorname{Mef} 2 d$ & CAGCAGCCACCTCAGCAACA & CCAAGTATCCAGCCGCATCC & NM_133665 \\
\hline$N r 2 e 3$ & TGCAGAATGAGCGCCAACCT & TGAAGAGCAGGCGAGCAGAT & NM_013708 \\
\hline $\mathrm{Nrl}$ & TGTCTGTGCGCGAGTTGAAC & AAGAGGTGTGTGTGGTCGTC & NM_008736 \\
\hline Rls & ACTGGACAGTTCGTGTACTG & GCTTCACTCGAGTCTTCTTG & NM_012053 \\
\hline
\end{tabular}


zerland) according to the manufacturer's instructions. Protein amounts in cytosolic and nuclear fractions were determined using the micro BCA protein assay (Thermo Fisher Scientific). Three mouse retinas were used for each condition studied.

\section{Western Blot Analysis}

Equal amounts of proteins, as determined by the microBCA protein assay (Thermo Fisher Scientific), were resolved on 10\% SDS-PAGE gels followed by transfer on PVDF membrane (Whatman/Schleicher \& Schuell, Sanford, UK). Membranes were blocked in 5\% nonfat dried milk before being immunoassayed using rabbit antibodies against Mef2c (diluted 1/400; Aviva Systems Biology, San Diego, CA) and high mobility group protein B1 (HMGB1; diluted 1/5,000; Abcam, Cambridge, UK), and a mouse antibody against tubulin (1/40,000; Sigma, Buchs, Switzerland). Mef2c blocking peptide (Aviva Systems Biology), used as immunogen to generate the Mef2c antibody, was used to assess the specificity of Mef2c immunoblotting. Briefly, primary Mef2c antibody was preadsorbed with a fivefold excess (weight) of blocking peptide in $500 \mu \mathrm{L}$ of blocking buffer before to be used in Western blot analysis.

\section{Cloning and Plasmids}

A 1311-bp fragment spanning from -1141 to +170 of the mouse Mef $2 c$ gene was amplified with primers mMef2cpfor (5'-GGGGTACCGATACTGGGTGATGCCATTC-3') and mMef2cprev (5'-CGGGATCCGCTTCTCCAGGAAGTTGTTC- $3^{\prime}$ ) and subcloned into the KpnI/BglII sites of the pGL2-promoter (pGL2-prom) luciferase reporter vector (Promega). This region contains the previously described myocytespecific enhancer and most of the first untranslated exon ${ }^{15}$ (see Fig. 7). $5^{\prime}$-promoter deletion constructs were amplified with forward primers mMef2cp-780for (5'-GGGGTACCCTTCCAACTGAATTTACTTA-3'), mMef2cp-714for (5'-CGGGGTACCTGTCCTTACCTTAACCAGTC-3'), mMef2cp-418for (5-GGGGTACCCAGTTTAATAACCTGAAATG-3'), respectively, and mMef2cprev. A KpnI-XbaI fragment spanning region $-1141 /-843$ was subcloned into a synthesis kit (pBluescriptII, pBSII; Stratagene, La Jolla, CA) and then transferred into the KpnI/SacI sites of pGL2-prom. A XbaI/HindIII fragment spanning region -843/-546 was subcloned into pBSII, excised with $\mathrm{KpnI} / \mathrm{BamHI}$ and subcloned into the KpnI/BglII sites of pGL2-prom. We cloned a 1171-bp fragment of the proximal promoter of the mouse $\operatorname{Rgr}$ (retinal G protein coupled receptor) gene, amplified with primers mRgrpfor (5'-GGGGTACCGGCATTGTTACAATTCCACC-3') and mRgrprev (5'-CGGGATCCAGCTCTTTTCTGGCTCTCTG-3') into the KpnI/BglII sites of the pGL2-prom. All constructs were verified by sequencing on a gene analyzer (ABI 3130xl Genetic Analyzer using the Big Dye Terminator Labeling Kit; Applied Biosystems, Carlsbad, CA). In addition, we used as luciferase reporter constructs a 225 -bp fragment of the bovine rhodopsin proximal promoter (BR225-Luc) and a 250-bp fragment of the mouse M-opsin proximal promoter (Mop250-Luc). ${ }^{33}$ The different expression vectors for mouse (m) Mef2c were pcDNA3.1-mMEF2C, pcDNA3.1-mMEF2C-VP16, where amino acids 1 to 143 were fused to the VP16 transcriptional activation domain, and the DNA-binding deficient dominant-negative pcDNA3.1-mMEF2C(p.R3T), bearing a mutation in the highly conserved N-terminal MADS-box. ${ }^{34}$ Finally, the previously described expression vectors for human (h) CRX (cone-rod homeobox), pcDNA3.1/HisC-hCRX, and for human NRL (neural retina leucine zipper), pMT-hNRL, were used. ${ }^{33}$

\section{Cell Culture and Luciferase Assays}

For transactivation assays, human embryonic kidney (HEK) 293T cells were grown at $37^{\circ} \mathrm{C}$ and $5 \% \mathrm{CO}_{2}$ in Dulbecco's modified Eagle's medium (DMEM; PAA Laboratories GmbH, Pasching, Austria) supplemented with $10 \% \mathrm{FCS}, 100 \mathrm{U} / \mathrm{mL}$ penicillin and $100 \mu \mathrm{g} / \mathrm{mL}$ streptomycin (Invitrogen). Cells were plated in 12-well plates (TPP, Trasadingen, Switzerland) and transfected at a confluence of $30 \%$ with the calcium phosphate method (ProFection; Promega). Per well, 30 ng of each of the expression vectors and $500 \mathrm{ng}$ of luciferase reporter constructs were used. As internal standard, 33 ng of plasmid $\operatorname{CMV} \beta$ (Clontech, Moutain View, CA) encoding $\beta$-galactosidase were used. To keep the total transfected DNA quantity constant, appropriate quantities of pcDNA3.1/HisC empty vector were added in all experiments. All plasmids were prepared on columns (NucleoBond PC500; MachereyNagel, Düren, Germany). Enzymatic activities were assessed with an assay system (Luciferase Assay System; Promega) and standard $\beta$-Gal assay. Two to four independent experiments were performed in triplicate $(n=6-12)$.

\section{In Silico Promoter Analysis}

Prediction for transcription factor binding sites was achieved both manually for A/T-rich sequences and with AliBaba 2.1, Match 1.0, and Patch 1.0 software (available at http://www.gene-regulation.com for c-Jun, c-Fos, c-Maf, Crx, and Pax response elements). oligonucleotide microarray
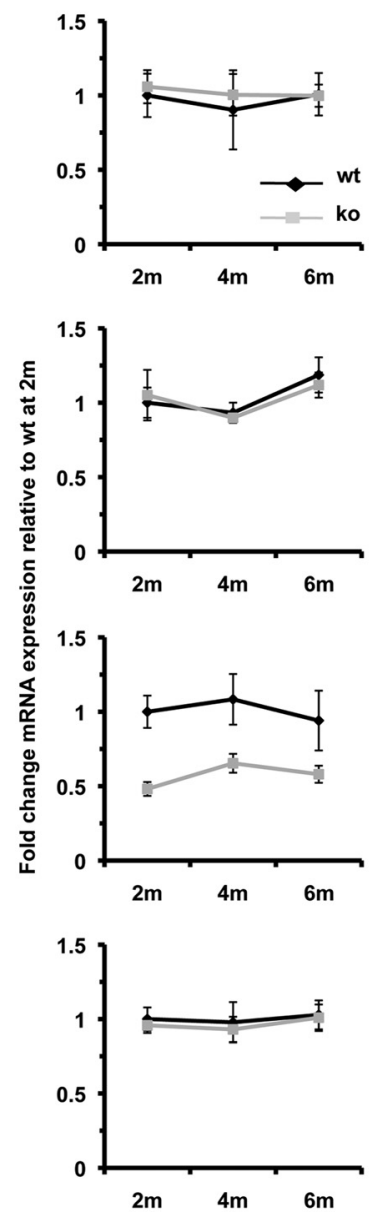

quantitative PCR
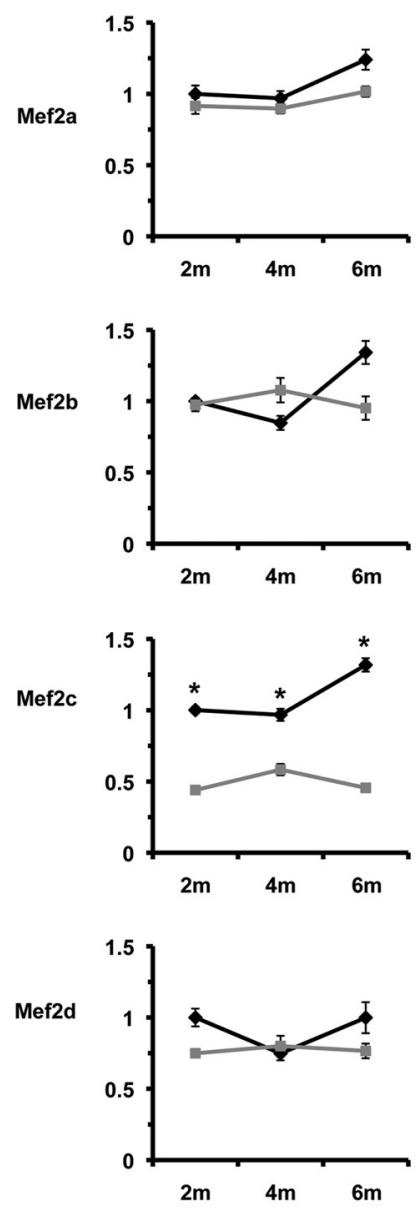

FIGURE 1. Transcriptional downregulation of Mef2c in Rpe65 $5^{-/-}$retinas. mRNA expression from wild-type (wt, black diamond) and Rpe $65^{-1-}$ retinas (ko, gray square) was assessed at 2,4 , and 6 months of age $(2-6 \mathrm{~m})$ by oligonucleotide microarray (left panels) and validated by quantitative PCR (right panels). Relative mRNA levels were expressed as fold inductions relative to samples from 2-month-old wt mice arbitrarily set to 1 . Mef2c was the only Mef 2 member markedly downregulated in diseased retinas at all ages, while expression of the other Mef2 members remained essentially unchanged in microarray and qPCR analyses. Data are the mean \pm SEM of three (left panels) and three to four (right panels) independent experiments. ${ }^{*} P<0.01$ by ANOVA test for Mef2c expression levels in $R p e 65^{-/-}$versus wt. 


\section{Statistical Analysis}

Statistical analysis was performed with commercially available software (Prism 4.0.2; GraphPad Software Inc., La Jolla, CA). All results were expressed as mean \pm SEM of the indicated number of experiments.

\section{Results}

\section{Downregulated Mef2c mRNA and Protein Expression in Rpe65 $5^{-/-}$Mice}

In an initial gene expression profiling study, we observed that 130 known genes were downregulated in $R p e 65^{-/-}$mice during disease progression. ${ }^{28}$ In this study, we investigated the retinal expression of Mef2 transcription factor members in 2-, 4-, and 6-month-old WT and Rpe $65^{-/-}$mice by oligonucleotide microarray and qPCR analyses. In the microarray study, Mef2c was the only member showing altered expression in $R p e 65^{-/-}$ retinas when compared to WT retinas. A 50\% decrease in Mef2c mRNA level was observed at all ages during disease progression, whereas Mef2a, -b, and -d mRNA expression remained unchanged (Fig. 1, left panels). QPCR confirmed the decreased level of Mef2c mRNA in Rpe $65^{-/-}$retinas compared to WT levels (Fig. 1, right panels).

The intracellular localization of Mef2c in retinal cells was further investigated by Western blot analysis in adult WT and Rpe $65^{-/-}$retinas. After subcellular fractionation of retinal cell extracts, cytosolic and nuclear-enriched fractions were immunoassayed using a Mef2c antibody. The protein was detected in the cytosol and the nucleus of the retinal cells (Fig. 2A) and reduction of Mef2c at the protein level was confirmed in Rpe $65^{-/-}$retinas (Fig. 2B). High mobility group protein $\mathrm{B} 1$ (HMGB1; P63158, www. uniprot.org) was used as a nuclear marker to assess the quality of the nuclear-enriched fractions. The specificity of Mef2c immunoblotting was further confirmed by preadsorbing Mef2c antibody with a blocking peptide used as immunogen to generate the antibody (Fig. 2C).

\section{Expression of Mef2c mRNA in Adult WT Retinas}

Although transcriptome analysis in rod-deficient $\mathrm{Rho}^{-/-}, \mathrm{Nrl}^{-/-}$, and $\mathrm{Crx}^{-1-}$ mice indirectly indicated that Mef2c might be a rod-enriched transcription factor, ${ }^{29-31}$ its expression in the retina has not yet been addressed.

We assessed Mef2c mRNA expression in 2-month-old WT retinas by in situ hybridization with antisense (Fig. 3A) and sense probes (Fig. 3B). Mef2c was expressed in the inner nuclear layer (INL), outer nuclear layer (ONL), and inner segments (IS) of the photoreceptors. Higher magnification of the antisense-probed retina section (Fig. 3E) revealed Mef2c mRNA expression in the cellular bodies and IS compartments of the photoreceptors. Mef2c is highly expressed in striated muscles; its expression in ocular muscle tissue hybridized with antisense (Fig. 3C) and sense (Fig. 3D) probes confirmed the specificity of the probe.

\section{Early Altered Regulation of Nr1, Nr2e3 and Mef2c Transcriptional Factors in Rpe $65^{-/-}$Mice}

In the microarray analysis, we observed that the expression of $\mathrm{Nrl}$ and Nr2e3 was downregulated in $R p e 65^{-1-}$ retinas at 2 to 6 months of age (Fig. 4). We addressed the question whether the expression of the rod-specific transcription factors and Mef2c might be altered during early postnatal $(\mathrm{P})$ retinal development from $\mathrm{P} 7$ to P13. As shown by qPCR analysis (Fig. 5), the expression of $\mathrm{Nrl}, \mathrm{Nr} 2 \mathrm{e} 3$, and $\mathrm{Mef} 2 \mathrm{c}$ was significantly decreased in $R p e 65^{-/-}$retinas from P13 onwards, at a time when photoreceptors are terminally differentiated. These observations demonstrate that the regulation of the rod-specific transcriptional network, along with Mef2c, is altered early in the newly differentiated retinas of $R p e 65^{-/-}$mice.

\section{Mef2c Acts as a Transcriptional Repressor in HEK293T Cells}

$\mathrm{Nrl}$ interacts with Crx and $\mathrm{Nr} 2 \mathrm{e} 3$ and regulates, either alone or synergistically, the expression of cone opsins ${ }^{35,36}$ and several rod-specific genes, including rhodopsin ${ }^{35-37}$ and rod cGMPphosphodiesterase. $^{38,39}$

We addressed the question whether Mef2c might regulate the expression of photoreceptor-specific genes, possibly in conjunction with other known rod-enriched transcription factors. We first tested the transcriptional activity of Mef2c on a
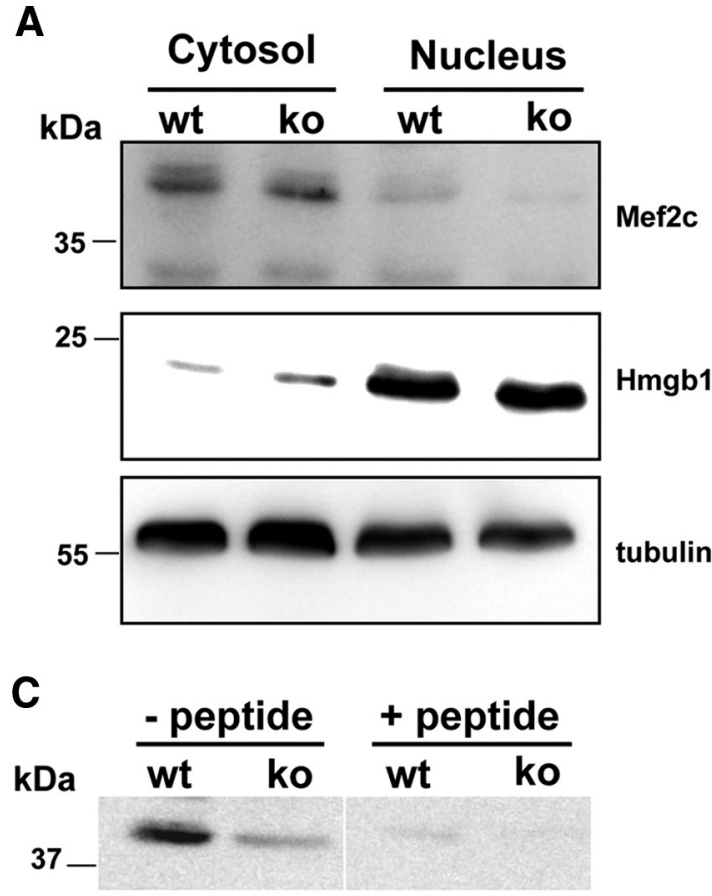

B

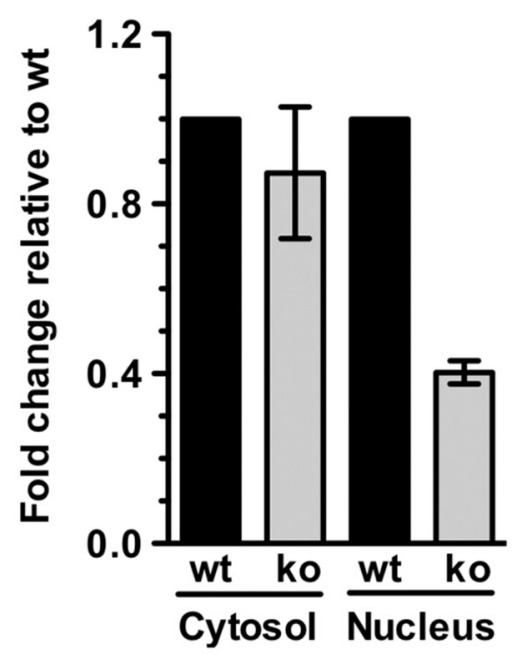

Figure 2. Western blot analysis of Mef2c protein in Rpe $65^{-/-}$retinas. After subcellular fractionation of retinal cell extracts from adult wt and $R p e 65^{-/-}$(ko) mice, $50 \mu \mathrm{g}$ of total proteins were loaded in 10\% SDS-PAGE and immunoassayed using antibodies against Mef2c and the nuclear marker HMGB1. (A) Mef2c protein was detected in the cytosol and nucleus of retinal cells. The decreased expression of Mef2c at the protein level was confirmed in $R p e 65^{-/-}$retinas. (B) After densitometric analyses of Mef2c and tubulin immunoblots, tubulin-normalized Mef2c was expressed as fold change relative to wt retinas. Data are the mean \pm SEM of three independent experiments. (C) Specificity of Mef2c immunoblotting was further confirmed by preadsorbing Mef2c antibody with a specific blocking peptide. 


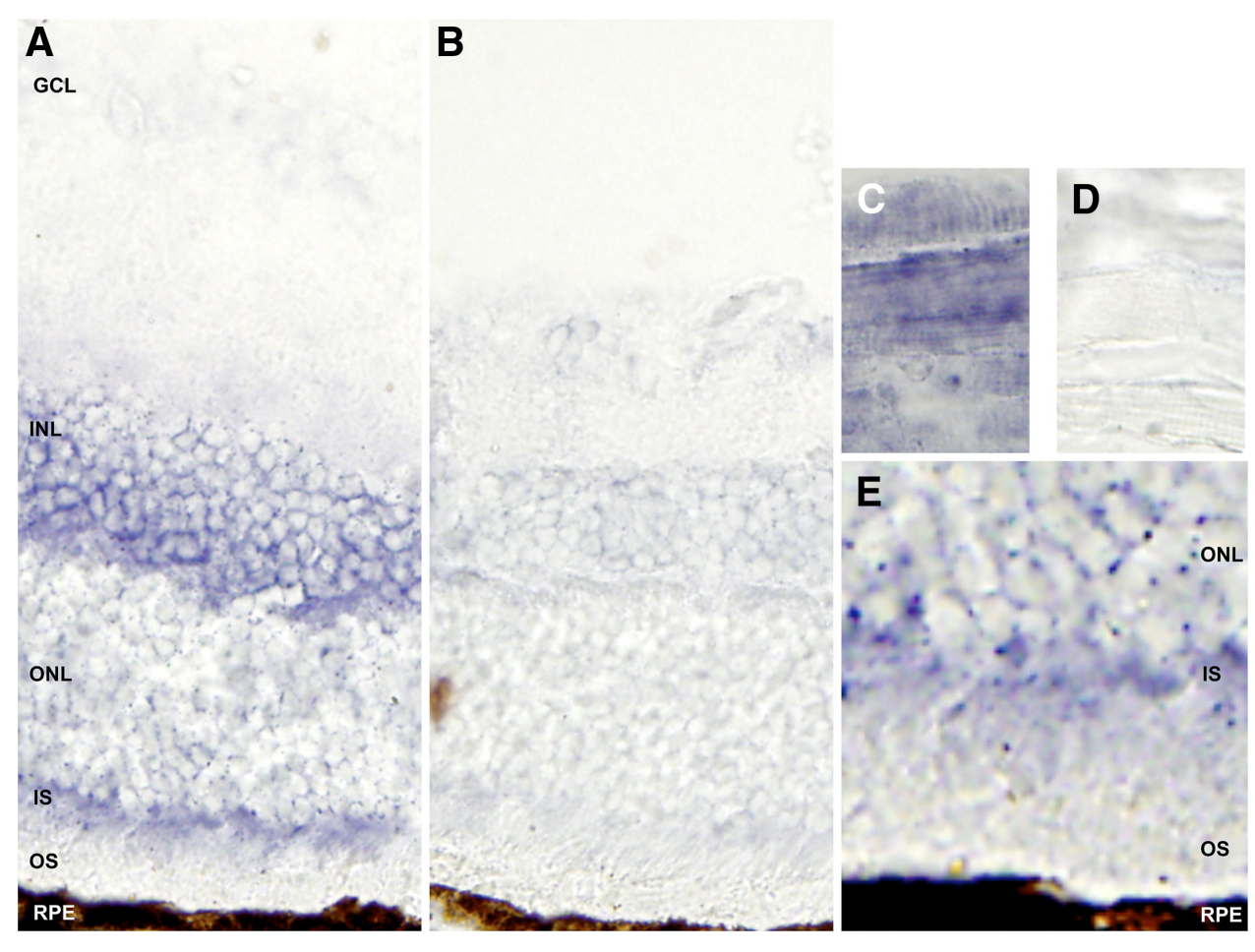

FIGURE 3. Localized expression of Mef2c in retinal cell layers. In situ hybridization studies were performed on retina sections from 2-month-old wt mice with antisense (A) and control sense (B) probes. Mef2c mRNA was detected in INL, ONL, and photoreceptor IS. As a control of the specificity of the probe, Mef2c transcript was observed in ocular muscle tissue hybridized with antisense probe (C) but not with sense probe (D). Higher magnification (E) of the antisenseexpression in the cellular bodies and the IS compartments of the photoreceptors. GCL, ganglion cell layer; INL, inner nuclear layer; IS, inner segments; ONL, outer nuclear layer; OS, outer segments. probed retina section reveals Mef2c

B

rod-specific rhodopsin promoter, and the cone-specific M-opsin promoter.

$-1141 /+170 \mathrm{Mef} 2 \mathrm{c}$ promoter region encompassing the previously described myocyte-specific enhancer, located upstream of the first untranslated exon of the mouse Mef2c gene. ${ }^{15}$ The 429 base pairs immediately located upstream of the transcription initiation site are evolutionarily-conserved among vertebrates, and Mef2c was unable to bind to A/T-rich sequences located in this region, even in presence of myogenins binding to a conserved E-box located at $-67 /-72 .{ }^{15}$ Mef2c repressed the activity of this myocyte-specific enhancer construct by $>60 \%$ (Fig. 6A). Repression was relieved in presence of the dominant-negative Mef2c, indicating that the repressor activity of Mef2c was partially DNA-binding dependent. As a positive control, we used a constitutively active Mef2c fused to the VP16 activator domain, which resulted in increased transcriptional activity of the Mef2c promoter. We then tested whether photoreceptor-specific transcription factors would be able to regulate this promoter region (Fig. 6B). NRL increased the activity of the Mef $2 c$ promoter by approximately 2.5 -fold, whereas CRX did not. Interestingly, Mef2c acted as a repressor in presence of NRL and/or CRX. This Mef2c repressor activity was also observed on the retina-specific RGR promoter, the

\section{NRL Stimulates Mef2c Promoter Activity}

To identify promoter regions mediating the regulation of Mef $2 c$ by Mef2c and NRL, we analyzed the sequence for potential transcription factor binding sites. We were unable to identify reported $\mathrm{Nrl}$ response element (NRE) sequences, such as an extended activator protein (AP)-1 binding site present in the rhodopsin promoter (TGCTGATTCCAGCC), or nonclassical binding sites present in the Nr2e3 (GCTGAGT/AAAG) and Ppp2r5c (protein phosphatase $2 \mathrm{~A}$ regulatory subunit) (GGCCAATCAGC) promoters. $^{37,40,41}$ We noticed a repetition of three A/T-rich sequences at locations $-758 /-749$ (CTAAAAAAGA), $-743 /-734$ (CTAAAAATTC), and $-729 /-720$ (CTAATAAATG). In silico analysis identified a c-Maf binding site at $-206 /-200$ (ATTTTCC) and, of note for retinal development, potential CRX, PAX6, and PAX2 response elements (Fig. 7A).

We generated a number of deletions of the Mef2c proximal promoter to evaluate the activity of the different transcription
Figure 4. Downregulated expression of rod-specific transcription factors during progression of the disease in $R$ pe $65^{-1-}$ mice. Microarray analysis in wt (black diamond) and Rpe $65^{-/-}$(ko, gray square) mice at 2,4 , and 6 months of age (2-6m) revealed that $\mathrm{Nrl}$ and $\mathrm{Nr} 2 \mathrm{e} 3$ expression was decreased in diseased retinas relative to wt retinas. Data are the mean \pm SEM of three independent experiments. ${ }^{*} P<0.01$ by ANOVA test for $\mathrm{Nrl}$ and Nr2e3 expression levels in Rpe65 $5^{-/-}$versus wt, except for Nr2e 3 at 4 months of age.
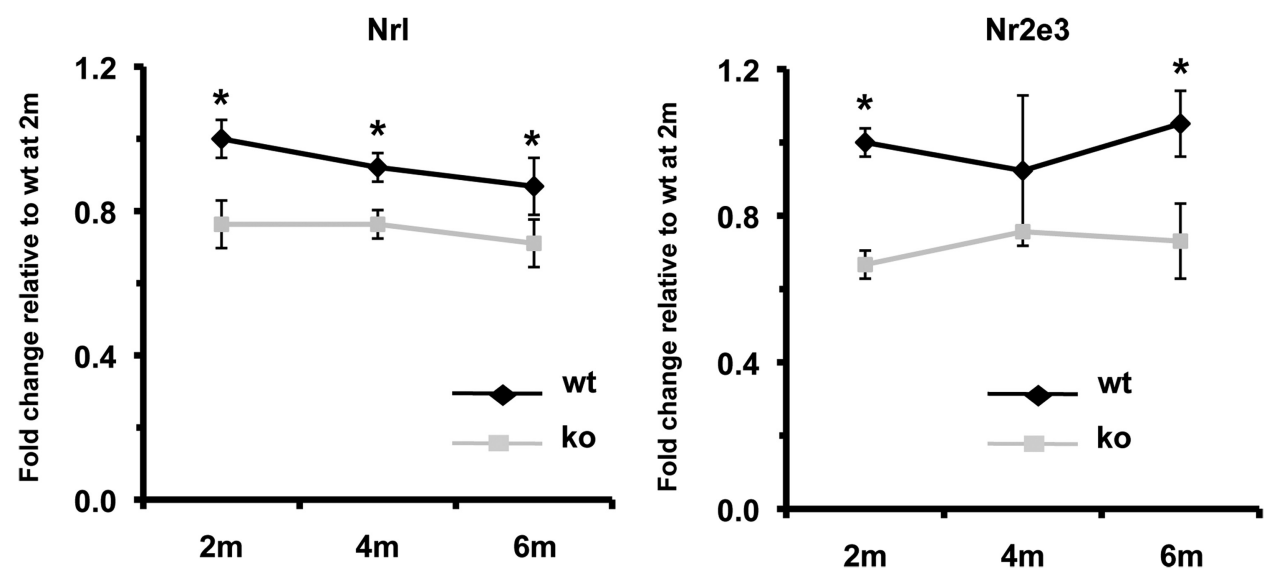

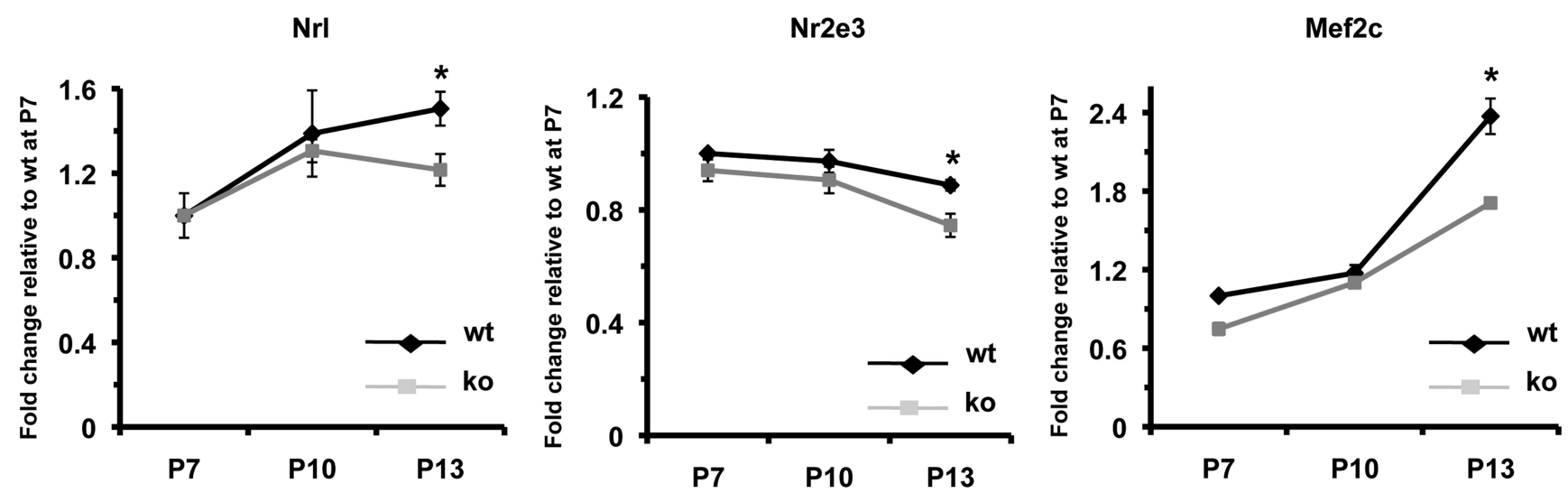

FiguRE 5. Early downregulation of photoreceptor-enriched transcription factors in $R p e 65^{-/-}$mice. qPCR analysis of the expression of Nrl, Nr2e 3 , and Mef2c during postnatal retinal development in wt (black diamond) and Rpe65 $5^{-1-}$ (ko; gray square) mice at P7, P10, and P13 expressed as fold change relative to wt at P7. Impaired expression of the three transcription factors was observed from P13 onwards in $R p e 65^{-1-}$ retinas. Data are the mean \pm SEM of four independent experiments. ${ }^{*} P<0.005$ by $t$-test for transcript expression levels at P13 in $R p e 65^{-/-}$versus wt.

factors (Fig. 7B). The longest fragment $(-1141 /+170)$ showed the highest activity (Fig. 7C), and NRL induced the promoter activity by 2.5 -fold. Whereas the activity of the $-1141 /-843$ was
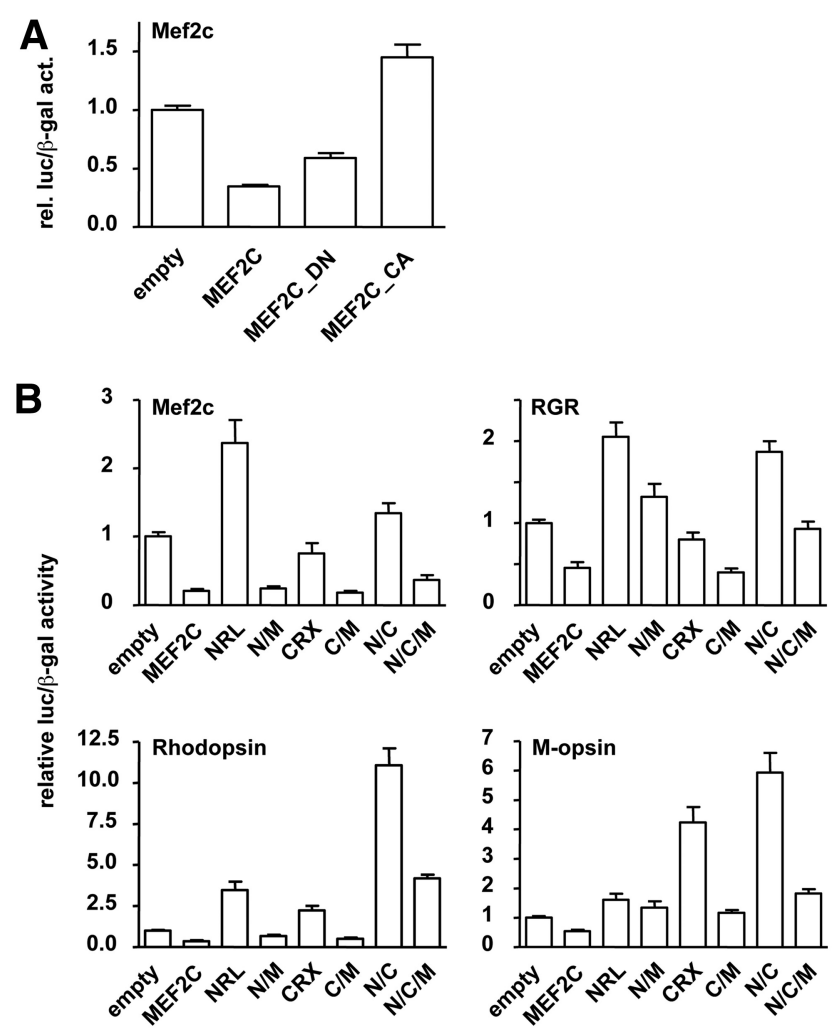

FIGURE 6. Transcriptional regulation by Mef2c in HEK293T cells. (A) Mef2c acts as a repressor on the $M e f 2 c$ myocyte-specific enhancer. Expression vectors for mouse wt Mef2c (MEF2C), a dominant-negative Mef2c (MEF2C_DN), and a constitutively active Mef2c (MEF2C_CA) were cotransfected with the mouse Mef2c luciferase reporter construct. (B) Mef2c acts as a repressor toward the photoreceptor-specific transcription factors NRL and CRX. Combinations of mouse MEF2C (M), human NRL (N), and human CRX (C) expression vectors were cotransfected with Mef2c, RGR, rhodopsin, or M-opsin luciferase reporter constructs. Three independent experiments were performed in triplicate $(n=9)$ and results are shown as mean \pm SEM. Firefly luciferase activities were normalized to $\beta$-galactosidase activities and presented as fold-inductions relative to the respective promoter construct cotransfected with the empty pcDNA3.1 expression vector. at the limit of detection, the promoter construct encompassing region $-843 /-546$ was induced 3.3-fold by NRL. Activation by NRL was 2.3 -fold with the $-780 /+170$ construct, but reduced to 1.4 - and 1.9 -fold with the $-714 /+170$ and $-418 /+170$ constructs. Mef2c acted as a repressor on all promoter deletion constructs. Despite the in silico prediction of two CRX response elements, CRX was unable to activate any of the promoter constructs.

\section{Discussion}

In the present study, we reported the expression of the Mef2 family of transcription factors in the retina. Among them, Mef2c was the only member showing marked downregulation at transcript and protein levels during disease progression in Rpe $65^{-1-}$ mice. In situ hybridization analysis revealed that retinal expression of Mef2c was restricted to the interneurons of the INL and to the cellular bodies and inner segments of the photoreceptors. Crx, Nrl, and Nr2e 3 showed a photoreceptorrestricted expression, with their RNAs being also present in the inner segments. ${ }^{42-44}$ IS-specific enriched RNA is a common pattern among the rod-enriched transcription factors and other photoreceptor-enriched genes, although the functional significance of this remains unclear. These data indicate that Mef2c displayed a similar expression pattern in the retina because it was observed for the rod-enriched transcriptional regulators directing photoreceptor development.

In this study, we reported altered expression of Mef2c early in the newly differentiated $R p e 65^{-/-}$retinas, which was concomitant with the decreased expression of $\mathrm{Nrl}$ and $\mathrm{Nr} 2 \mathrm{e} 3$. In the initial microarray experiment, 213 genes were also downregulated in Rpe65-deficient retinas. ${ }^{28}$ Several transcriptional regulators were present among these, including HIF $1 \alpha$ (hypoxia inducible factor 1 , alpha subunit), Pbx1 (pre B-cell leukemia transcription factor 1), $\operatorname{PPAR} \alpha$ (peroxisome proliferatoractivated receptor alpha). Their potential interaction with Mef2c, or involvement in Mef2c-dependent pathways, remains to be determined. Mef2c retinal expression has been shown to be almost completely abolished in $\mathrm{Crx}^{-/-30} \mathrm{Nrl}^{-/-30,31}$ and $\mathrm{Rbo}^{-/-29}$ mice, suggesting that Mef2c may be a rod-enriched transcription factor. Moreover, Mef2c was identified as a putative direct target of the rod-specific Nrl transcription factor, indicating that Mef2c may be involved in the transcriptional network directing photoreceptor differentiation and maintenance. ${ }^{31}$ In addition, we observed in healthy retina that Mef2c transcriptional expression, similarly to what is known for $\mathrm{Nrl}$ 
A

-1141 GATACTGGGTGATGCCATTCAGGTCTATGTTTTATCTGAAAGTGGAGCCCTGCAGGGAAR -1081 TGAGGCTTGTTGAGTGGCTGTTTAGACATAGTAAGTTAATAGGAACCTTGTTTATTCTAC -1021 AAAGCATTTTTATTTTGACCCAGTTTCTTTCTCTCATCACACCCAAGCACTGTAAACAGC -961 AATAGTATGGTAGAACAGGTACTTTTTGCAACTCCCGTTAACTTCACTTAAGAAATCATG -901 ACTGCCAAAGTGGAGTCTTACAAGATTTTGTCCTAGCATACTCCTTCTTAGCACGCTTTC -841 TAGAGTTGGACTGTTAAATTTGTGCCAGATACATCAATGAATACCGCCTGCCTATCTTTT -781 TCTTCCAACTGAATTTACTTATCTAAAAAAGAAATCGTCTAAAAATTCACCTGCTAATAA -721 ATGTAAATGTCCTTACCTTAACCAGTCATTAAGGAATACCAGCTAAATCAGGGTCACACA -601 CAATCGTTTAAGTGCCATGACCATCCAGTTTTGACACCGAGTCTCTTAGAGTTACAAGCT -541 TTCTAATTTGGGAGCATGATTAATCCCTCCTATGTGATAAGTTTTAACCTTCTAATATTT -481 CTTTGGATTGAAAAAAGCAAATGAGCTGCGGCAAAGAATGGCAAATAACTACAGTGCTTA C -361 AGCCACAATTAATTGAACAAAAACTTGTCTTGTTCCAAGATTATTCTTGGAAATGTAATT -301 TTAAAGCCTGTGTGAAATGAGGAAACTTAACTTTTTATACCATATGAAAGCAATTTCATT -241 TTTTAGGAATGATTTTGGATAGACTTCCGATTGGATATTTTCCATTGGAACTAACAGTG' -121 GGTTGTCAATGATACCTTTACAGCTAAATTTACTCCAGAGTGACATGAACAGGTGCACCC -61 TGGCCTGCCAGACACTTGTGCAGAGGGATCACGCATCTCACCGCTTGACGATCAAGGGGC CAAAGCTTCGGTGTTCATAGAAMAgGAGAGGAgGCGAGCGAGCCCAAACTGGGGGTTI CTCTTCAAAGCCAGCTGGTCTGGCTTTATTCTACAGGAATTTTTTTACCTGTCAGAGTTT

+120 GGACAACAAAGCCCTCAGCAGGTGCTGACGGGAACAACTTCCTGGAGAAGCAGAAAGGCA
+180 CTGGTGAGTTTCAATTGCCAAAAAATATGTACTTTTTTTAAATCTCTAAAAGTTGATTTC

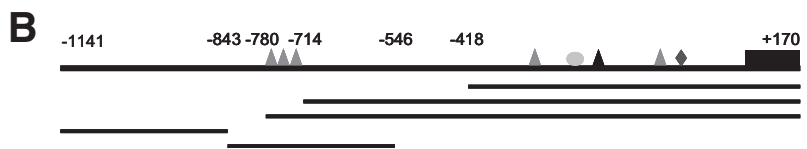

C

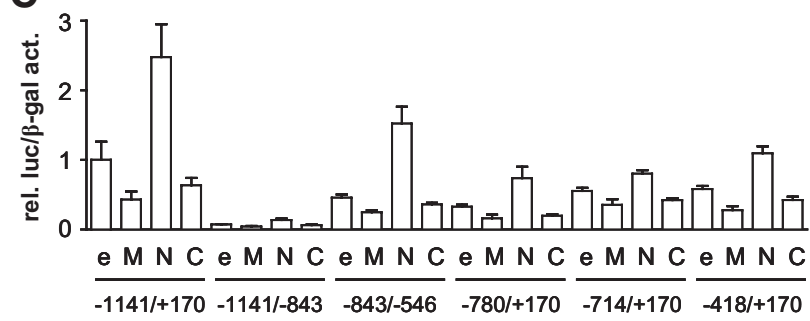

FIGURE 7. NRL activates the mouse Mef2c promoter. (A) Sequence of the analyzed region of the mouse Mef2c proximal promoter, located upstream of the first untranslated exon of Mef2c (bold, bighlighted) and containing a myocyte-specific enhancer conserved among vertebrates $(-429 /+1)$. A/T-rich sequences (bold, underlined) and potential response elements for c-Maf (bold, bigbligbted, underlined), Crx (italics), Pax6 (underlined), Pax2 (bighlighted), and an E-box motfif binding MyoD (bold, bighlighted) are indicated. (B) Schematic representation of the Mef2c promoter constructs used in this study. On top, the nucleotide numbering is indicated, with +1 being the transcription start site. The first exon is represented as a black box. A/T-rich regions (light gray triangle), c-Maf binding site (black triangle), Pax6 binding site (oval), and E-box (diamond) are indicated for clarity. (C) Deletion analysis of the Mef2c promoter by transactivation assays in HEK293T cells. Nucleotide numbering of the Mef2c regions subcloned into the luciferase reporter constructs are indicated at the bottom. Mouse $\operatorname{Mef} 2 \mathrm{c}(\mathrm{M})$, human NRL $(\mathrm{N})$, and human CRX (C) expression vectors were cotransfected with the luciferase reporter constructs $(n=6-12)$. Firefly luciferase activities were normalized to $\beta$-galactosidase activities and presented as fold-inductions relative to the $-1141 /+170$ promoter construct cotransfected with the empty pcDNA3.1 expression vector (e).

and rod-specific genes, increased during maturation of the photoreceptors from P13 to P30.

In our heterologous transactivation assay in $293 \mathrm{~T}$ cells, NRL alone was able to activate $M e f 2 c$ expression. NRL is essential for rod development, ${ }^{45}$ and Mef2c might therefore be important for the development and maintenance of fully functional rods. Notably, CRX, a master regulator of photoreceptor development, ${ }^{42}$ was unable to activate Mef2c expression. This suggested that Mef2c might function after terminal differentiation of cone and rod photoreceptors has been initiated. Interestingly, we have recently identified $M e f 2 c$ as a target of the nuclear receptor NR1D1 in the adult mouse retina, ${ }^{46}$ and
NR1D1 was able to activate the Mef2c proximal promoter in 293T-cell based transactivation assays to a similar extent than NRL (P. Escher and Neena Haider, unpublished data). Given that these studies have been performed in a heterologous cell system, the physiological relevance needs to be ascertained. However, these different experimental observations provided circumstantial evidence that Mef2c is involved rod photoreceptor maintenance.

From a mechanistic point of view, it is important to remember that no consensus NRL binding sites have been identified in the Mef2c proximal promoter, and that Mef2c, NRL, and other Maf-box containing transcription factors might compete for the same binding sites. This could also contribute to the understanding of previously reported conflicting results about regulation of the Mef2c promoter by Mef2c, in conjunction with E-box binding myogenin transcription factors. ${ }^{15,47}$

Despite the fact that Mef2c had primarily been reported to be a transcriptional activator, ${ }^{12}$ it appeared to be a transcriptional repressor on all retina-specific promoter fragments tested so far. Interestingly, in the olfactory system, another sensory system, Mef2c has been shown to be necessary to repress the neuronal gonadotropin-releasing hormone gene. ${ }^{48}$ With respect to repression of the Mef2c promoter by Mef2c, a similar autoregulation, either repressing or activating, had been shown for Mef2a. ${ }^{49}$ Reportedly, conversion of Mef2c into a repressor can be mediated by sumoylation. ${ }^{50,51}$

These data support the view that Mef2c is part of a Nrldependent rod-specific transcriptional network, may act as a transcriptional repressor of photoreceptor-specific genes and may impact on the development and maintenance of photoreceptors.

\section{Acknowledgments}

The authors thank Sylviane Métrailler and Nathalie Voirol for their expert technical assistance; Shiming Chen for pcDNA3.1/HisC-hCRX, pMT-NRL, BR225-Luc, and Mop250-Luc plasmids; Eric Olson for pcDNA3.1-mMEF2C; and Jeffery D. Molkentin for pcDNA3.1mMEF2C(R3T) and -mMEF2C-VP16 expression plasmids. The RGR promoter construct was generated for a collaborative project with Neena Haider.

\section{References}

1. El Matri L, Ambresin A, Schorderet DF, et al. Phenotype of three consanguineous Tunisian families with early-onset retinal degeneration caused by an R91W homozygous mutation in the RPE65 gene. Graefes Arch Clin Exp Opbthalmol. 2006;244:1104-1112.

2. Jacobson SG, Aleman TS, Cideciyan AV, et al. Identifying photoreceptors in blind eyes caused by RPE65 mutations: prerequisite for human gene therapy success. Proc Natl Acad Sci US A. 2005;102: 6177-6182

3. Jin M, Li S, Moghrabi WN, Sun H, Travis GH. Rpe65 is the retinoid isomerase in bovine retinal pigment epithelium. Cell. 2005;122: 449-459.

4. Moiseyev G, Chen Y, Takahashi Y, Wu BX, Ma J-X. RPE65 is the isomerohydrolase in the retinoid visual cycle. Proc Natl Acad Sci U S A. 2005;102:12413-12418.

5. Moiseyev G, Takahashi Y, Chen Y, et al. RPE65 is an iron(II)dependent isomerohydrolase in the retinoid visual cycle. $\mathrm{J} \mathrm{Biol}$ Chem. 2006;281:2835-2840.

6. Redmond TM, Yu S, Lee E, et al. Rpe65 is necessary for production of 11-cis-vitamin A in the retinal visual cycle. Nat Genet. 1998;20: 344-351.

7. Galvin J, Fishman G, Stone E, Koenekoop R. Clinical phenotypes in carriers of Leber congenital amaurosis mutations. Ophthalmology. 2005;112:349-356.

8. Seeliger M, Grimm C, Stahlberg F, et al. New views on RPE65 deficiency: the rod system is the source of vision in a mouse model of Leber congenital amaurosis. Nat Genet. 2001;29:70-74. 
9. Woodruff ML, Wang Z, Chung HY, Redmond TM, Fain GL, Lem $\mathrm{J}$. Spontaneous activity of opsin apoprotein is a cause of Leber congenital amaurosis. Nat Genet. 2003;35:158-164.

10. Hamann S, Schorderet DF, Cottet S. Bax-induced apoptosis in Leber's congenital amaurosis: a dual role in rod and cone degeneration. PLOS ONE. 2009; 4:e6616.

11. Black BL, Olson EN. Transcriptional control of muscle development by myocyte enhancer factor-2 (MEF2) proteins. Annu Rev Cell Dev Biol. 1998;14:167-196.

12. Potthoff MJ, Olson EN. MEF2: a central regulator of diverse developmental programs. Development. 2007;134:4131-4140.

13. Molkentin JD, Black BL, Martin JF, Olson EN. Mutational analysis of the DNA binding, dimerization, and transcriptional activation domains of MEF2C. Mol Cell Biol. 1996;16:2627-2636.

14. Lin Q, Schwarz J, Bucana C, Olson EN. Control of mouse cardiac morphogenesis and myogenesis by transcription factor MEF2C. Science. 1997;276:1404-1407.

15. Dodou E, Xu S-M, Black BL. mef2c is activated directly by myogenic basic helix-loop-helix proteins during skeletal muscle development in vivo. Mech Dev. 2003;120:1021-1032.

16. Heidt $\mathrm{AB}, \mathrm{Black} \mathrm{BL}$. Transgenic mice that express Cre recombinase under control of a skeletal muscle-specific promoter from mef2c. Genesis. 2005;42:28-32.

17. Martin JF, Schwarz JJ, Olson EN. Myocyte enhancer factor (MEF) 2C: a tissue-restricted member of the MEF-2 family of transcription factors. Proc Natl Acad Sci US A. 1993;90:5282-5286.

18. Edmondson DG, Lyons GE, Martin JF, Olson EN. Mef2 gene expression marks the cardiac and skeletal muscle lineages during mouse embryogenesis. Development. 1994;120:1251-1263.

19. Lyons GE, Micales BK, Schwarz J, Martin JF, Olson EN. Expression of mef 2 genes in the mouse central nervous system suggests a role in neuronal maturation. J Neurosci. 1995;15:5727-5738.

20. Heidenreich KA, Linseman DA. Myocyte enhancer factor-2 transcription factors in neuronal differentiation and survival. $\mathrm{Mol} \mathrm{Neu}$ robiol. 2004;29:155-166.

21. Leifer D, Krainc D, Yu YT, et al. MEF2C, a MADS/MEF2-family transcription factor expressed in a laminar distribution in cerebral cortex. Proc Natl Acad Sci U S A. 1993;90:1546-1550.

22. Li H, Radford JC, Ragusa MJ, et al. Transcription factor MEF2C influences neural stem/progenitor cell differentiation and maturation in vivo. Proc Natl Acad Sci US A. 2008;105:9397-9402.

23. Mao Z, Bonni A, Xia F, Nadal-Vicens M, Greenberg ME. Neuronal activity-dependent cell survival mediated by transcription factor MEF2. Science. 1999;286:785-790.

24. Engels H, Wohlleber E, Zink A, et al. A novel microdeletion syn drome involving 5q14.3-q15: clinical and molecular cytogenetic characterization of three patients. Eur J Hum Genet. 2009;17: 1592-1599.

25. Le Meur N, Holder-Espinasse M, Jaillard S, et al. MEF2C haploin sufficiency caused by either microdeletion of the $5 q 14.3$ region or mutation is responsible for severe mental retardation with stereotypic movements, epilepsy and/or cerebral malformations. $J$ Med Genet. 2010;47:22-29.

26. Novara F, Beri S, Giorda R, et al. Refining the phenotype associated with MEF2C haploinsufficiency. Clin Genet. 2010;78:471-477.

27. Zweier M, Gregor A, Zweier C, et al. Mutations in MEF2C from the 5 q14.3q15 microdeletion syndrome region are a frequent cause of severe mental retardation and diminish MECP2 and CDKL5 expression. Hum Mutat. 2010;31:722-733.

28. Cottet S, Michaut L, Boisset G, Schlecht U, Gehring W, Schorderet DF. Biological characterization of gene response in Rpe $65^{-1-}$ mouse model of Leber's congenital amaurosis during progression of the disease. FASEB J. 2006;20:2036-2049.

29. Kennan A, Aherne A, Palfi A, et al. Identification of an IMPDH1 mutation in autosomal dominant retinitis pigmentosa (RP10) revealed following comparative microarray analysis of transcripts derived from retinas of wild-type and Rho(-/-) mice. Hum $\mathrm{Mol}$ Genet. 2002;11:547-557.

30. Hsiau THC, Diaconu C, Myers CA, Lee J, Cepko CL, Corbo JC. The cis-regulatory logic of the mammalian photoreceptor transcriptional network. PLOS ONE. 2007;2:e643.
31. Yoshida S, Mears AJ, Friedman JS, et al. Expression profiling of the developing and mature $\mathrm{Nrl}^{-/-}$mouse retina: identification of retinal disease candidates and transcriptional regulatory targets of $\mathrm{Nrl}$. Hum Mol Genet. 2004;13:1487-1503.

32. Braissant O, Wahli W. Differential expression of peroxisome proliferator-activated receptor-alpha, -beta, and -gamma during rat embryonic development. Endocrinology. 1998;139:2748-2754.

33. Peng G-H, Ahmad O, Ahmad F, Liu J, Chen S. The photoreceptorspecific nuclear receptor $\mathrm{Nr} 2 \mathrm{e} 3$ interacts with Crx and exerts opposing effects on the transcription of rod versus cone genes. Hum Mol Genet. 2005;14:747-764.

34. Xu J, Gong NL, Bodi I, Aronow BJ, Backx PH, Molkentin JD Myocyte enhancer factors $2 \mathrm{~A}$ and $2 \mathrm{C}$ induce dilated cardiomyopathy in transgenic mice. J Biol Chem. 2006;281:9152-9162.

35. Escher P, Gouras P, Roduit R, et al. Mutations in NR2E3 can cause dominant or recessive retinal degenerations in the same family. Hum Mutat. 2009;30:342-351.

36. Roduit R, Escher P, Schorderet DF. Mutations in the DNA-binding domain of NR2E3 affect in vivo dimerization and interaction with CRX. PloS ONE. 2009; $4: \mathrm{e} 7379$.

37. Rehemtulla A, Warwar R, Kumar R, Ji X, Zack DJ, Swaroop A. The basic motif-leucine zipper transcription factor $\mathrm{Nrl}$ can positively regulate rhodopsin gene expression. Proc Natl Acad Sci USA. 1996;93:191-195.

38. Lerner LE, Gribanova YE, Ji M, Knox BE, Farber DB. Nrl and Sp nuclear proteins mediate transcription of rod-specific cGMP-phosphodiesterase beta-subunit gene: involvement of multiple response elements. J Biol Chem. 2001;276:34999-35007.

39. Pittler SJ, Zhang Y, Chen S, et al. Functional analysis of the rod photoreceptor cGMP phosphodiesterase alpha-subunit gene promoter: $\mathrm{Nrl}$ and $\mathrm{Crx}$ are required for full transcriptional activity. J Biol Chem. 2004;279:19800-19807.

40. Oh EC, Cheng H, Hao H, Jia L, Khan NW, Swaroop A. Rod differentiation factor NRL activates the expression of nuclear receptor NR2E3 to suppress the development of cone photoreceptors. Brain Res. 2008;1236:16-29.

41. Kim JW, Jang SM, Kim CH, An JH, Kang EJ, Choi KH. Neural retina leucine-zipper regulates the expression of $\operatorname{Ppp} 2 \mathrm{r} 5 \mathrm{c}$, the regulatory subunit of protein phosphatase 2A, in photoreceptor development. FEBS Lett J. 2010;277:5151-5160.

42. Furukawa T, Morrow EM, Cepko CL. Crx, a novel otx-like homeobox gene, shows photoreceptor-specific expression and regulates photoreceptor differentiation. Cell. 1997;91:531-541.

43. Blackshaw S, Harpavat S, Trimarchi J, et al. Genomic analysis of mouse retinal development. PLoS Biol. 2004;2:E247.

44. Blackshaw S, Fraioli RE, Furukawa T, Cepko CL. Comprehensive analysis of photoreceptor gene expression and the identification of candidate retinal disease genes. Cell. 2001;107:579-589.

45. Mears AJ, Kondo M, Swain PK, et al. $\mathrm{Nrl}$ is required for rod photoreceptor development. Nat Genet. 2001;29:447-452.

46. Mollema NJ, Yuan Y, Jelcick AS, et al. Nuclear receptor Rev-erb alpha (Nr1d1) functions in concert with Nr2e3 to regulate transcriptional networks in the retina. PLOS ONE. 2011;6: e17494.

47. Wang DZ, Valdez MR, McAnally J, Richardson J, Olson EN. The Mef2c gene is a direct transcriptional target of myogenic bHLH and MEF2 proteins during skeletal muscle development. Development. 2001; 128:4623-4633.

48. Allen MP, Xu M, Zeng C, Tobet SA, Wierman ME. Myocyte enhancer factors-2B and -2C are required for adhesion related kinase repression of neuronal gonadotropin releasing hormone gene expression. J Biol Chem. 2000;275:39662-39670.

49. Ramachandran B, Yu G, Li S, Zhu B, Gulick T. Myocyte enhancer factor $2 \mathrm{~A}$ is transcriptionally autoregulated. J Biol Chem. 2008; 283:10318-10329.

50. Shalizi A, Gaudillière B, Yuan Z, et al. A calcium-regulated MEF2 sumoylation switch controls postsynaptic differentiation. Science. 2006;311:1012-1017.

51. Kang J, Gocke CB, Yu H. Phosphorylation-facilitated sumoylation of MEF2C negatively regulates its transcriptional activity. BMC Biochem. 2006;7:5. 